\title{
Regional response to large-scale emergency events: Building on historical data
}

\author{
Carol Romanowski, ${ }^{a 1}$ Rajendra Raj,${ }^{a}$ Jennifer Schneider,${ }^{b}$ Sumita Mishra,${ }^{c}$ \\ Vinay Shivshankar, ${ }^{a}$ Srikant Ayengar ${ }^{a}$ and Fernando Cueva ${ }^{a}$ \\ ${ }^{a}$ Department of Computer Science, Rochester Institute of Technology, 1 Lomb Memorial \\ Drive, Rochester, New York 14623, USA \\ ${ }^{b}$ Collaboratory for Resilience and Recovery, Rochester Institute of Technology, 1 Lomb \\ Memorial Drive, Rochester, New York 14623, USA \\ ${ }^{c}$ Department of Computing Security, Rochester Institute of Technology, 1 Lomb \\ Memorial Drive, Rochester, New York 14623, USA
}

\begin{abstract}
A widespread emergency event in the United States triggers the activation of a regional emergency operations center that manages a coordinated response to the disaster. Historically, the time-critical decisions made by emergency managers in the face of incomplete information and inadequate historical emergency event data have been guided primarily by their experience. The learning curve for emergency managers, especially novice managers, is steep, and is exacerbated by the complexity and scope of emergency events. This paper proposes a methodology designed to provide emergency managers with locality-specific information and resource allocation recommendations for large-scale event response, creating the foundation for a decision support system that draws on emergency event data. This work is the first to use locally-specific data for an emergency management decision support system. Two major allocation scenarios that influence the number of resources allocated to an event are considered and solutions are suggested to address them. Although the methodology is developed for a mid-sized region, it is generalizable to any region.
\end{abstract}

\footnotetext{
${ }^{1}$ Corresponding author: Carol Romanowski (cjrcms@rit.edu)
} 


\section{Keywords}

Emergency Management; Large-Scale Events; Historical Data Analytics; Data Mining; Resource Allocation

Submitted: January 11, 2015

Revision: April 19, 2015

Accepted: July 10, 2015

\section{Introduction}

When an emergency occurs that is beyond the capability of a single incident commander to handle, control of the response is usually assumed by a centralized emergency operations center that operates under a unified command system. Several characteristics of centralized response management make regional large-scale emergency (disaster) management a difficult, if not wicked, problem. First, efficient emergency operations rely on effective resource allocation under severe time constraints. Second, mid-sized (one to five million persons) and smaller regions rely heavily on volunteer responders and are typically resource-constrained. Third, the emergency manager and the emergency operations center must integrate multiple heterogeneous data streams arriving at varying rates, often resulting in incomplete, possibly incorrect, knowledge of the scope of the disaster. Finally, the emergency manager's personal experience with local crisis management heavily influences the overall effectiveness of the response. These characteristics highlight the need for a robust decision support system (DSS) for regional emergency management that is built on local historical data and incorporates crucial information about the critical infrastructure assets in the region of interest.

Emergency management and incident command rely heavily on information and experience to implement an effective response. As disasters inherently combine repeated elements (historical experience) with new information, experienced emergency managers quickly categorize repeated elements and focus on novel aspects of the information flow, thus speeding up decision making. Inexperienced emergency managers, however, cannot efficiently triage the information, which often delays critical decisions.

The difference between a veteran emergency manager and a novice manager is experience; but the basis of all decision making, no matter the skill level, is relevant and usable information transformed from the available raw 


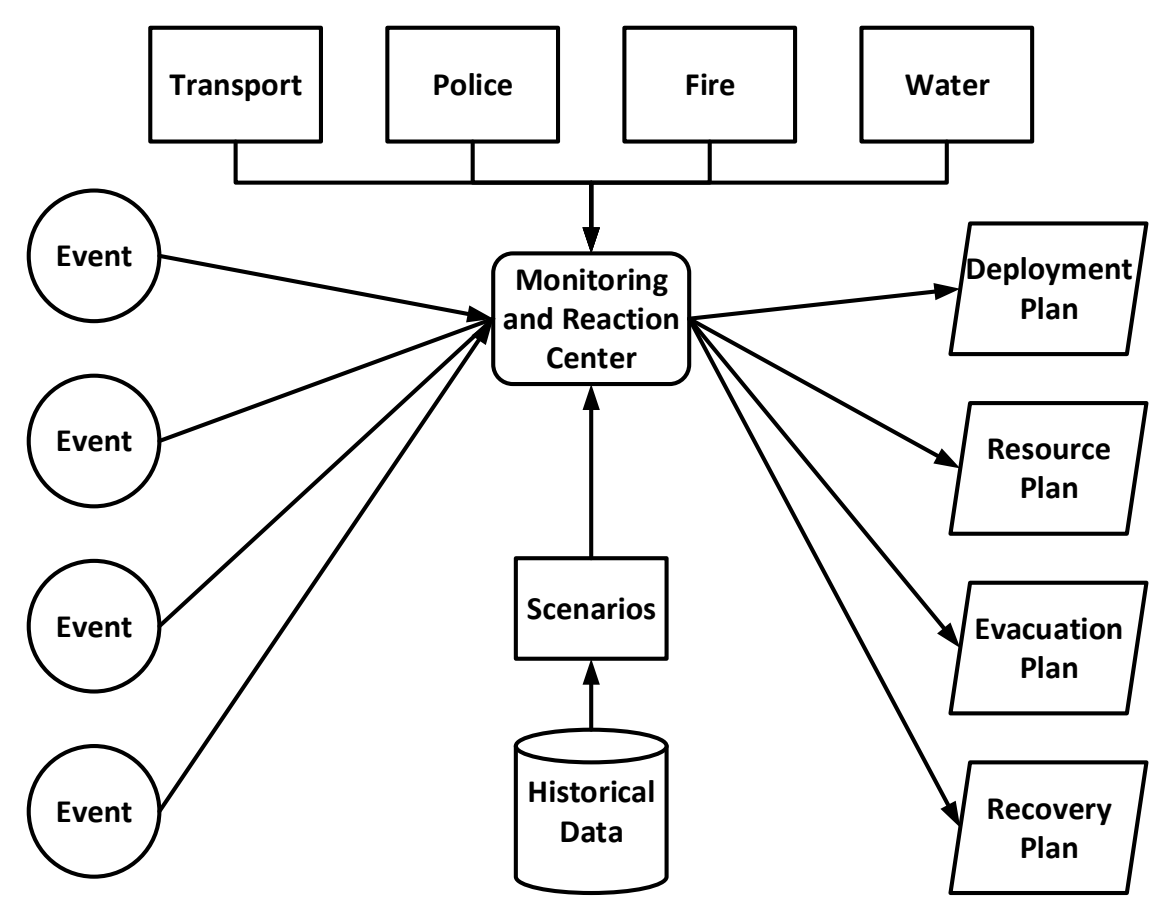

Figure 1: Role of historical data in emergency management decision support.

data. If a novice emergency manager could access this information and be guided by it, the playing field between veteran and novice becomes more level and the learning curve for the new emergency manager flattens out.

So where does the needed information come from? All the municipalities in the United States or Canada with 911 systems (and equivalent systems in other countries) possess enormous amounts of locally-specific data on emergency events in their regions. This data includes - at minimum - what, where and when events occurred; their durations; the resources used in event response; and the timelines of actions during the active response periods. If this data can be mined effectively, the historical experience of many responders, beyond that of a single individual, can be replicated.

Figure 1 shows how historical data can contribute to a decision support system for regional disaster response. The system receives real-time data from an event along with monitored data from first responders such as police and fire stations, and public utilities such as water and transportation. This data is sent to a central monitoring and reaction center (MRC), where 
it is aligned, integrated and fused with scenarios based on historical data. The monitoring and reaction center then provides recommendations to the emergency manager on the appropriate action plans for responding to events.

This paper focuses on the historical data and scenarios portion of the system shown in Figure 1. It examines issues involved in mining historical data for decision support, presents a methodology for using the data in regional response and discusses decision making in two distinct multi-nodal scenarios.

\section{Decision support systems in emergency management}

This section provides an overview of the use of decision support systems [11] in emergency management and examines some of the related work.

In the United States, the Department of Homeland Security (DHS) coordinates homeland protection. Created in 2002 from 22 existing agencies, the U.S. Department of Homeland Security has paid special attention to the integration of communications systems and processes, such as the National Incident Management System [10] and Tactical Interoperable Communication Planning [7], particularly during large-scale regional emergency responses that involve multiple agencies. Communications is the foundation for coordinated information flows and information flows serve as the foundation for situational awareness and decision making.

A variety of approaches have been adopted to incorporate decision support systems in emergency management. A typical decision support system divides information into processes, units and events using three main components: data management, model management and dialogue management [15]. As information flows and requests for information from senior decision makers and field operations increase, a decision support system can perform prioritization and pre-sorting during the information gathering phase, helping increase the speed of the remaining cycles in the process. Bryant [3] has presented a decision making model that attempts to reflect how a decision maker's past experience colors not just the observations, but also the perception of the current situation. Harrald and Jefferson [12] have explored enhancements to technology and computing software that can facilitate the incorporation of real-time data into processes, which is especially appropriate for emergency response.

Emergency management decision making has increasingly attempted to integrate information as a crisis unfolds, thereby enhancing situational awareness. "Boots on the ground" situational awareness models exist for integrat- 
ing real-time information in support of decision making $[5,12]$. However, additional work is needed to bridge the gap between these broad-based theoretical models and field-deployable implementations of decision systems.

Khouj et al. [14] have presented an innovative Monte-Carlo-based estimation system for making intelligent decisions after a disaster. They applied machine learning in an urban scenario involving a simulated earthquake in an urban community model comprising nine interdependent critical infrastructure cells. Their simulation focused on maximizing the number of patients discharged from two hospitals in the aftermath of an infrastructure disruption. Cavallini et al. [4] have described a decision support tool being developed under the Critical Infrastructure Simulation of Advanced Models for Interconnected Network Resilience (CRISADMIN) Project. Among other things, the project examines inter-organizational response, communications and coordination during emergencies.

Peng et al. [17] have developed an incident management decision support system with three components: a data processing tool that presents heterogeneous data in a unified format, a data mining tool that identifies patterns and anomalies, and a multi-criteria decision-making module that relies on inputs from the other two components. The approach of Peng and colleagues focuses on decision processes, not the creation of robust inputs needed to support quality decision making. Also, their approach was tested on agro-meteorological events that lack the immediacy needs of emergency management.

Other authors $[13,23]$ point to the usefulness of data-mined mobile and social network platforms and other rapidly-evolving data to assist with emergency response and recovery. In fact, several volunteer and endorsed systems (e.g., CrisisMappers [6]) are specifically designed for this purpose. However, although these systems are useful, they focus on community or victim perspectives instead of responder or decision-maker perspectives, and are not retrospective in nature. This work focuses on the first phase of community resilience, not the overall resilience.

\section{Decision support with local data}

Regional response decisions require two foundational pieces. First, the emergency manager needs a comprehensive database of critical infrastructure/key resources $(\mathrm{CI} / \mathrm{KRs})$ in the region. Second the emergency manager needs to know how these assets are interrelated. 
$\mathrm{CI} / \mathrm{KRs}$ are assets that are essential to maintain national security, national public health or both [2]. A nationally-rated CI/KR meets the minimum criteria of cost, lives lost or disrupted in the long term, and time to recover if the asset is compromised. In the context of a mid-sized region, which is the focus of this work, CI/KRs may or may not be nationally rated as critical, but are certainly regionally rated as such. Generating and maintaining a complete list of $\mathrm{CI} / \mathrm{KRs}$ in the metropolitan statistical area (MSA) of a mid-sized region is a challenge because they require deep knowledge of the surrounding counties. For example, the metropolitan statistical area of Rochester, New York includes its central county of Monroe and four adjacent counties (Orleans, Livingston, Ontario and Wayne). Within this metropolitan statistical area are dozens of small cities, towns and villages, each with its own critical infrastructure.

The U.S. Department of Homeland Security developed the Automated Critical Asset Management System (ACAMS), a comprehensive database that focused on physical security of the built environment while ignoring other important aspects such as cyber infrastructure and human capital [8]. Nevertheless, ACAMS served as a starting point for smaller metropolitan statistical areas to catalog their CI/KRs. However, after the demise of ACAMS and its replacement by the Infrastructure Protection Gateway [9], smaller metropolitan statistical areas need both a new data management structure and the technological skills to fully integrate $\mathrm{CI} / \mathrm{KR}$ information into regional responses.

An additional concern with $\mathrm{CI} / \mathrm{KRs}$ is the risk posed by the dependence of one asset on the operational capacity of another asset. For example, traffic signals are dependent on power; firefighting depends on the availability of water. As Figure 2 shows, dependencies can cause cascading risk, where the loss of one asset results in the degradation or loss of other assets. Inbound risk is where the loss of an asset outside the region impacts an asset inside the metropolitan statistical area. Outbound risk is where the loss of an asset in a metropolitan statistical area causes an operational disruption outside the area.

In the Monroe-Rochester metropolitan statistical area, as in several other New York counties and elsewhere, the regional emergency operations center uses a web-based board system to manage event data. However, this system is not real-time, does not push information automatically to users and is difficult and expensive to customize. Ideally, CI/KR data would be integrated with such a system and the emergency manager would be alerted when an 


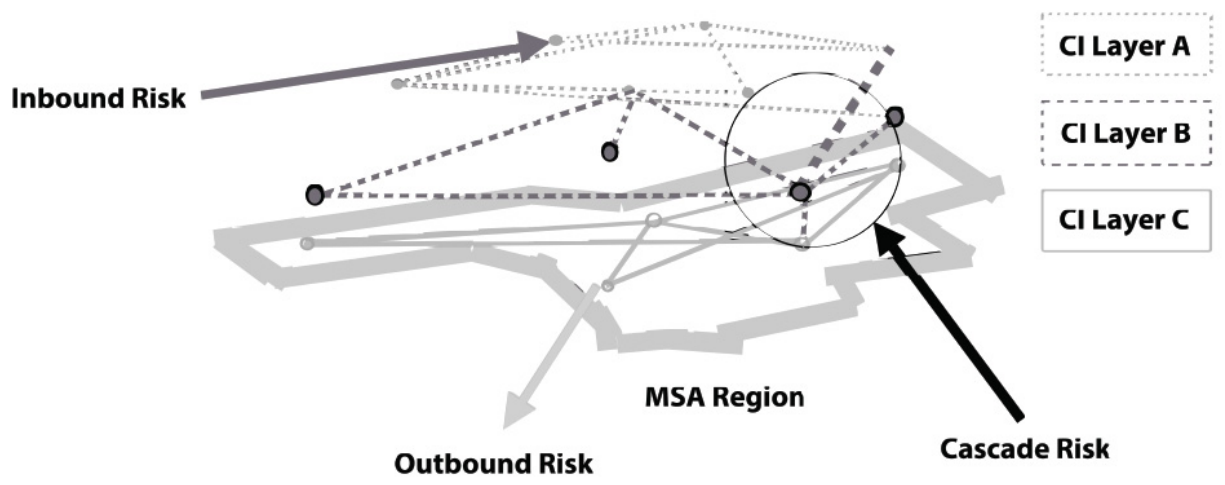

Figure 2: Risks arising from critical infrastructure dependencies.

asset is in danger of being compromised by a disaster event.

Major confidentiality issues arise when using $\mathrm{CI} / \mathrm{KR}$ data because most of the information is voluntarily submitted by private companies and is designated as protected critical infrastructure information (PCII) by the U.S. federal government. This designation limits data access to registered and trained PCII users, which has implications when modeling locally-specific systems.

The second foundational piece needed for effective regional response is a good understanding of how an event is expected to unfold over time. For mid-sized metropolitan statistical areas, disasters such as hurricanes, floods, chemical spills and fires are much more probable than terrorism incidents, which means that historical data on such disasters is available and can be used to build an incident footprint for regionally-common disasters. Note that the term "footprint" covers characteristics such as the time-based sequence of events, physical scope of a disaster, expected regional impact (physical and human) and expected resources allocated to deal with events.

The development of a footprint requires sufficient historical data for the identification and analysis of common regional events. The largest repository of historical disaster data is usually a 911 center, which handles emergency response data, and is the source for emergency event data for many of the agencies involved in regional emergency management. 911 centers in United States are required to store data for seven years; therefore, while the number of instances of particular events such as superstorms may be limited, other events are often well represented. 
Table 1: Main steps in the methodology.

\begin{tabular}{cl}
\hline Step & Description \\
\hline 1 & Data preparation and cleansing \\
2 & Identification of common regional events jointly with domain experts \\
3 & Attribute selection jointly with domain experts \\
4 & Incident footprinting \\
5 & Resource allocation prediction for separable events (additive model) \\
6 & Resource allocation prediction for inseparable events (potentiated model) \\
7 & Resource prediction refinement based on demonstrated impact on CI/KRs \\
\hline
\end{tabular}

\subsection{Steps in the methodology}

Historical data is a crucial element when designing a methodology for regional emergency response. This research effort was provided access to seven years (2005-2012) of 911 data calls in Monroe County. The dataset contained more than 8 million case records and 100 million associated "continuation records" corresponding to transcripts of communications that took place during the events. This data, supplemented with other publicly-available datasets, was used to generate incident footprints and to make resource allocation predictions.

Table 1 lists the main steps in the proposed methodology. The first three steps (data preparation and cleansing, identification of common regional events and attribute selection) are data- and region-specific and are not be covered in great detail here. The primary purpose of these preliminary steps is to produce a clean dataset that accurately represents the sources of disaster risk in a region.

\subsection{Generating an incident footprint}

The fourth step, incident footprinting, may be accomplished in many ways. Clustering was the most logical choice for the dataset used in this research. Clustering involves searching for natural groupings in data using methods such as $k$-means and expectation maximization. After the natural groupings are found, the event records contained in each cluster are analyzed to determine the distinct characteristics of the cluster.

As an example, consider a structural fire - an event that is common to all metropolitan statistical areas and one that can become a large regional disaster. Attributes of interest in characterizing fires are the time of year, time 


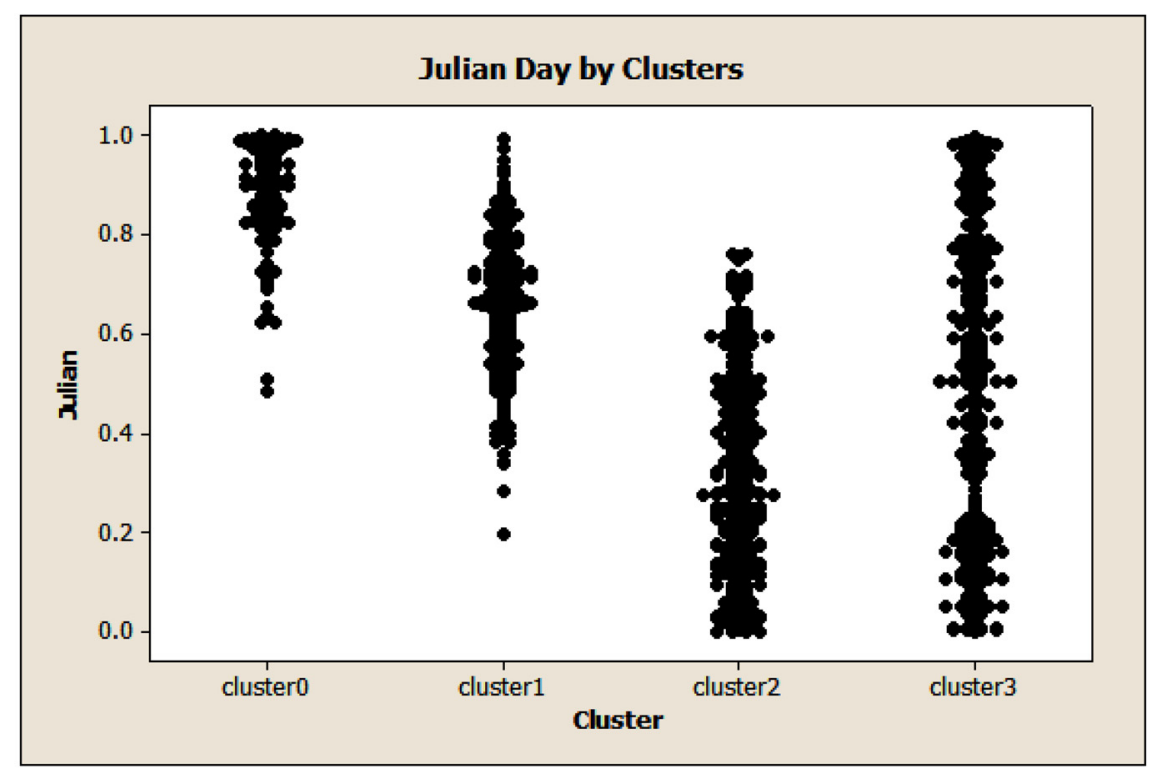

Figure 3: Clusters versus the Julian day.

of day, weather conditions, jurisdiction where the fire started, percentage of vacant structures in the area, percentage of older structures in the area and population density at the site of the event. Given these attributes, $k$-means clustering was performed on a dataset of 1,041 large structural fires. Four distinct clusters were identified. Figure 3, which presents a plot of the four clusters against the Julian day, shows clear differences between the groups for this particular attribute.

After the clusters were created, a generic footprint was constructed for each cluster type by examining the records classified as belonging to each cluster. Cluster 3, for example, represents fires in densely populated areas with older housing and high vacancy rates. In the case of these fires, mutual aid was summoned within 30 minutes of the first alarm and the mean length of incidents was 165 minutes, with a much smaller standard deviation than the other three clusters. The event records, along with their continuation records, can help determine how many (and which) jurisdictions were involved in responding to an event, the number and type of units sent to the scene, the times when the units were sent, which non-governmental organizations (such as the Red Cross) were present and when they arrived, and other characteristics such as the need for salt trucks in winter. This footprint 


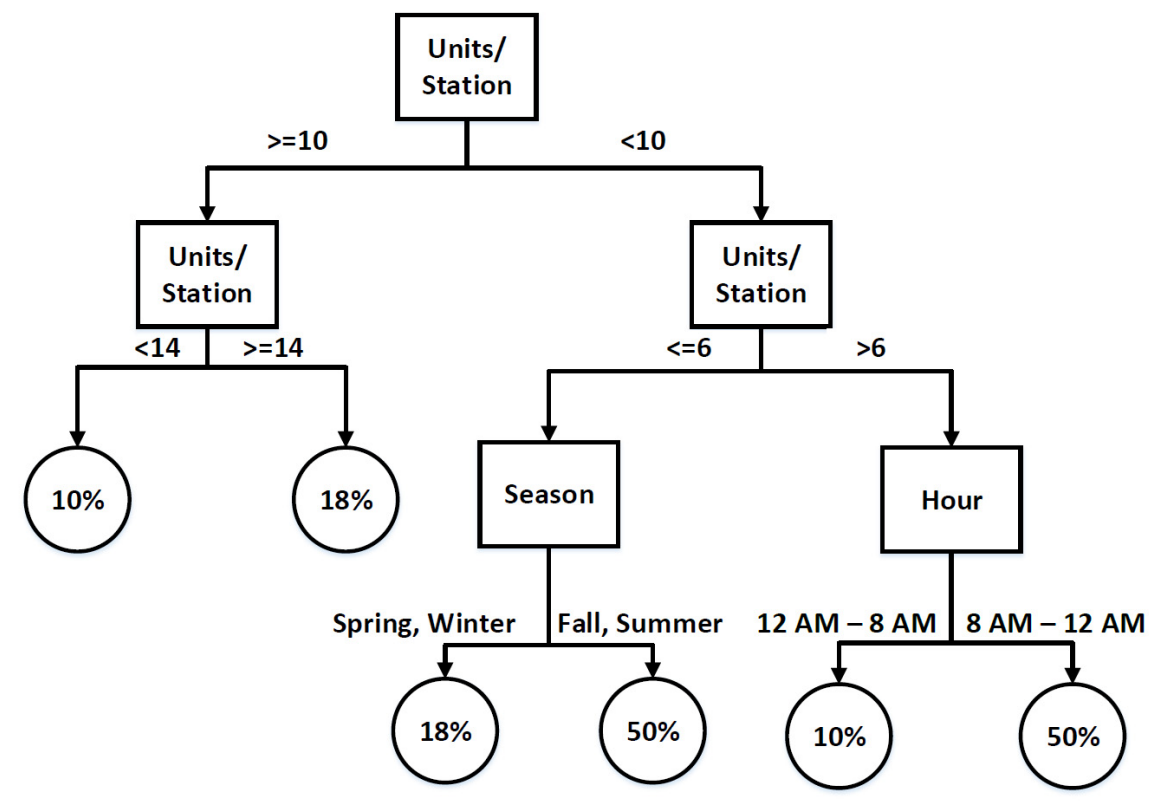

Figure 4: Predicting maximum resources for large fire events.

serves as the basis for several predictive efforts, from resource allocation to proactive resource staging and preemptive activities.

Figure 4 shows an example of resource prediction. The figure presents a simple decision tree generated from a dataset of 662 large structural fires that occurred in Monroe County between 2010 and 2012. Note that "large" denotes fires that took more than one hour to resolve and required aid from adjacent jurisdictions.

The root node of the tree is the number of units per fire station. For large stations with more than ten units, the fires used $10-18 \%$ of the available resources. For smaller stations, the time of day and season of the year had a large impact on the resources needed to fight fires. This result can be explained, in part, by the mostly volunteer crews at smaller stations; the difference in seasons is an empirical finding in this dataset based on the number of fires and their severity at different times of the year. A decision tree generated by data from a region other than Monroe County would look different, but the methodology for producing the tree from historical data would be the same.

The decision tree predicts the maximum resources, but not when the re- 


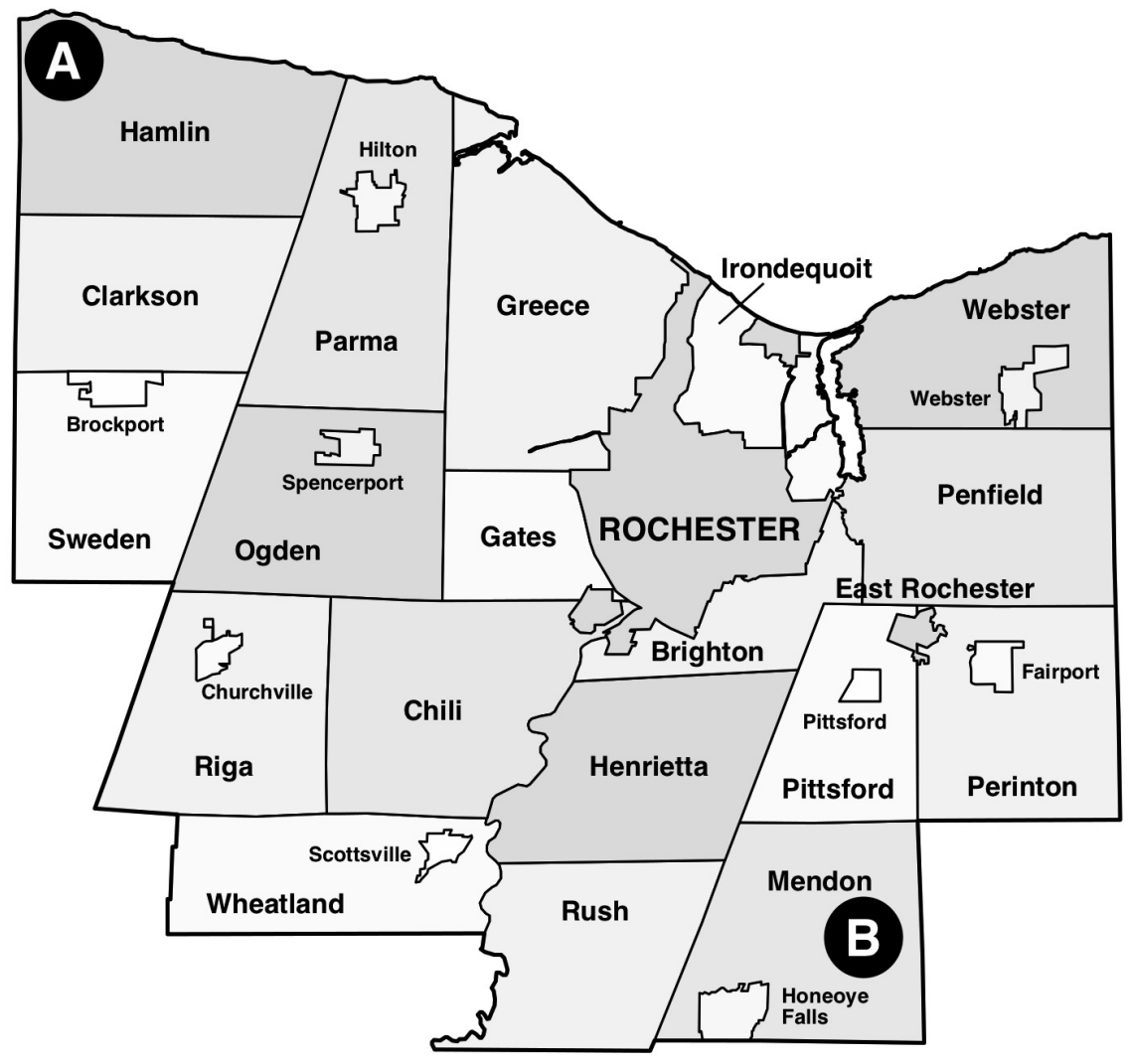

Figure 5: Additive scenario with concurrent events in non-contiguous jurisdictions.

sources need to be deployed. A general incident timeline helps an emergency manager visualize the expected demand for resources over the duration of an event.

\subsection{Predicting resource allocation}

At the emergency manager level, resource allocation prediction is dependent on the type of scenario. Two basic allocation scenarios are identified: (i) additive; and (ii) potentiated. In the additive scenario, large events occur simultaneously (or concurrently) in non-adjacent jurisdictions. For example, Figure 5 shows a map of Monroe County, New York [16] with two fire districts. Event A occurs in a fire district in the county's northwest corner. Event B occurs in a different fire district in the county's southeast corner. 


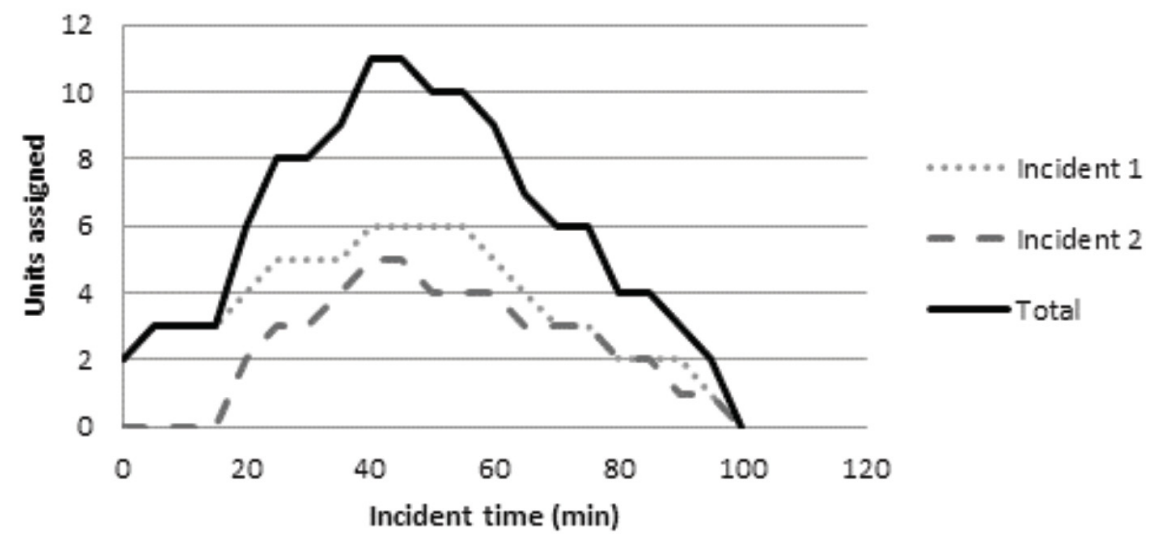

Figure 6: Resource needs in an additive scenario.

Because the events do not pull from the same pool of first responders, the number of resources needed to respond to each event is additive.

Figure 6 tracks the resources allocated for each event and their totals over the duration of the incident. The following equation is used to determine the number of resources needed in the additive scenario:

$$
R_{\text {final }}=R_{\text {predA }}+R_{\text {predB }}
$$

where $R_{\text {final }}$ is the total number of resources needed, $R_{\text {predA }}$ is the number of resources needed for Event $\mathrm{A}$ and $R_{\text {predB }}$ is the number of resources needed for Event B.

The second allocation scenario, the potentiated model, is much more difficult to manage. In this scenario, events occur simultaneously or concurrently, but in either the same or adjacent jurisdictions. The resources needed to respond to each event come from the same pool of available first responder units and, thus, the effect of a disaster is no longer additive. Figure 7 shows the scenario with concurrent Events A and B occurring in the same or contiguous jurisdictions. Figure 8 shows the gap between the additive scenario resource needs (sum (additive total) line) and the potentiated scenario needs (potentiated total line). Competition for the same resources, the greater need for unit coordination and the constrained capability of the region relative to the size of the total event are the main drivers behind the multiplicative nature of this scenario. Indeed, in the case of a potentiated event, $2+2$ may actually equal 40 . 


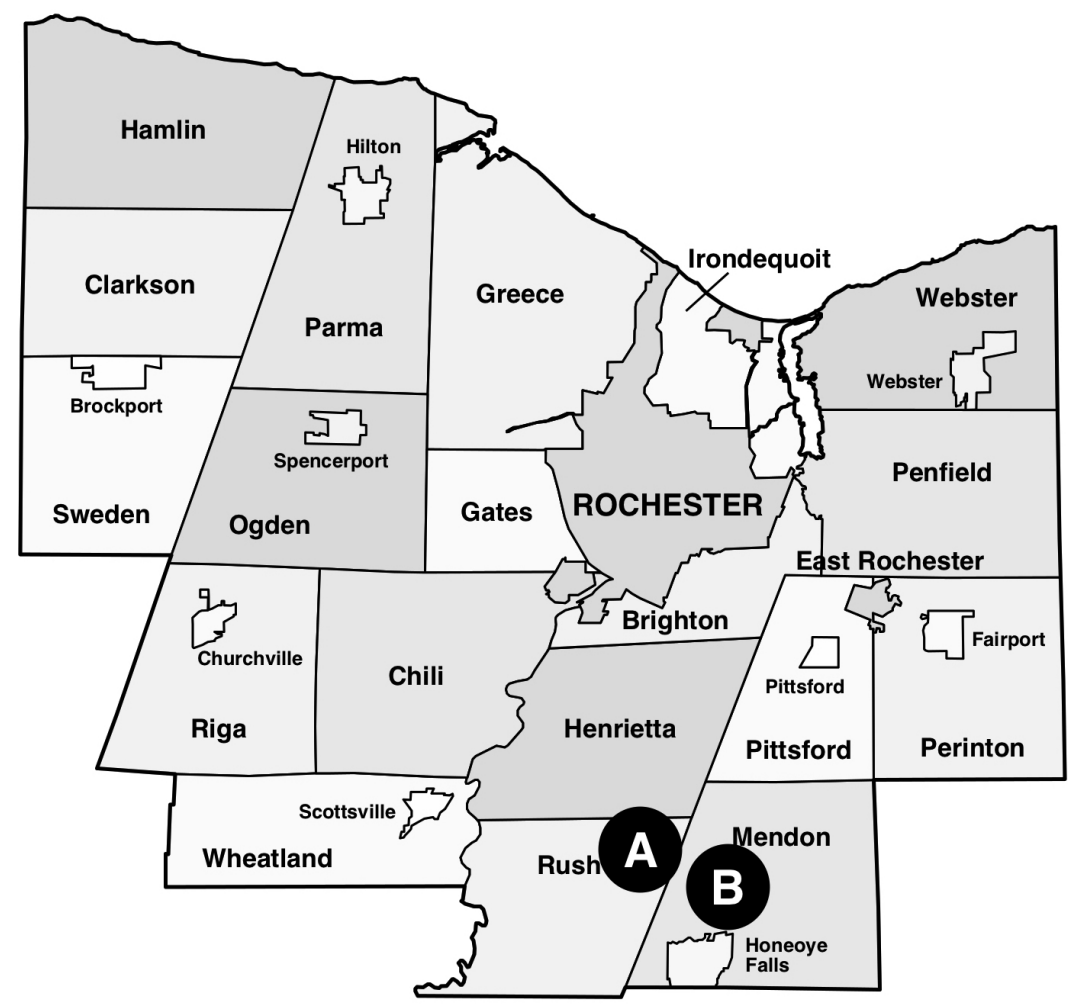

Figure 7: Potentiated scenario with concurrent events in contiguous jurisdictions.

Computing the resource needs for this scenario requires an adjustment of Equation (1) as follows:

$$
R_{\text {potentiated }}=(1+\beta) R_{\text {final }}
$$

where $R_{\text {potentiated }}$ is the recommended allocation, $R_{\text {final }}$ is calculated using Equation (1) and $\beta$ is a multiplier.

In the example in Figure 8, the multiplier $\beta$ was assigned a value of 0.6. However, in practice, the value of $\beta$ - and possibly even its range - are unknown. One way to determine a reasonable estimate of $\beta$ is to analyze several potentiated incidents and calculate the value of $\beta$ empirically. The dataset used in this research contained only one such incident - an arson/ambush in West Webster, New York that took place in 2012 [18]. The event started out as a car and house fire and involved an active shooter who killed two firefighters, wounded two more and left an entire neighborhood in 


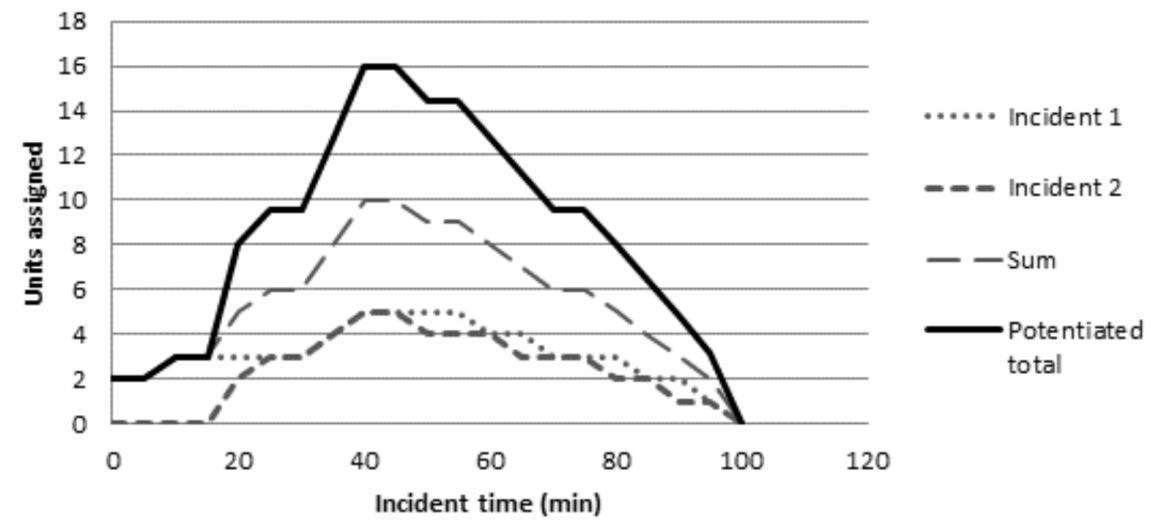

Figure 8: Resource needs in a potentiated scenario $(\beta=0.6)$.

ashes. The arson/ambush incident involved at least 52 units (some accounts list as many as 70), including New York State Police, Border Patrol, selfdeployed first responders, off-duty police and response units from all over the region. In contrast, an earlier fire in the same neighborhood around the same time of the year in 2010 required only four responder units. In order to calculate $\beta$ for the West Webster event, information is needed about the resources used in an active shooter incident, but this information is not present in the dataset. The size of the West Webster event illustrates the major challenges faced by emergency managers in potentiated scenarios.

\subsection{Integrating critical assets}

To this point, the presence of CI/KRs in a disaster event has not been considered. A large-scale event has the potential to destroy, damage or degrade $\mathrm{CI} / \mathrm{KR}$ in the immediate vicinity and cause cascading risk and outbound risk outside the event area. For this reason, emergency managers need to know which $\mathrm{CI} / \mathrm{KRs}$ are endangered by an event, the resources needed to protect the assets or prevent further damage and the assets that are dependent on the affected $\mathrm{CI} / \mathrm{KRs}$.

A CI/KR repository [8, 9] contains this information, which can be displayed to an emergency manager graphically on a map of the event area as well as via a dashboard view of the textual information. The decision maker then must determine the actions that must be taken to protect the threatened assets, prevent further damage to the assets that are already compromised or mitigate any further impact. 
The following equation is used to quantify the impact of a critical asset [19]:

$$
\varphi \sum_{\forall I}\left(P_{I}+C I_{I}+E_{I}\right)
$$

where $\varphi$ is the aggregate relative probability of occurrence (natural and intentional), $P_{I}$ is the population density impact, $C I_{I}$ is the number of critical infrastructure assets impacted and $E_{I}$ is the aggregate economic impact. While Equation (3) helps in risk management, it cannot be directly used to refine resource allocation recommendations. However, it does provide an approach to integrate asset protection in the calculations.

When CI/KRs are considered in resource allocation, two additional parameters are needed: (i) $\alpha$, the recommended number of resources for a particular $\mathrm{CI} / \mathrm{KR}$ type; and (ii) $\lambda$, which is equal to zero if the event distance from the asset is greater than a threshold distance $x$, and is equal to one otherwise. The value of $\alpha$ is dependent on the type of asset. A chemical plant requires different numbers and types of response resources than a hospital. The threshold value $x$ used to determine $\lambda$ is dependent on the event type. For example, a power substation located one mile away is of less concern than one that is within 500 feet of an active blaze.

The term $\varphi$ can be dropped for an event that has already occurred. The economic and population impact values can be used as weights to determine which $\mathrm{CI} / \mathrm{KRs}$ are critical if resources do not allow all the assets to be maximally protected.

The modified predicted resources for each event $R_{\text {pred }}$ is given by:

$$
R_{\text {pred }}=R_{\text {pred }}+\sum_{i} \alpha_{i} \lambda_{i}
$$

where $i$ is the number of CI/KRs impacted by the event.

In order to understand this modification, consider a situation where a fire has occurred and the maximum number of units predicted $R_{\text {pred }}$ is 7 . If a power substation is within 200 feet of the event, which is less than the $500 \mathrm{ft}$. threshold distance $x$ set for a fire event, then $\lambda=1$. Also, $\alpha=3$ responders would normally be assigned to the power substation. Assume that a telecommunications node is also within the threshold distance and $\alpha=2$ for this asset. In this case, $R_{\text {pred }}$ is computed as:

$$
R_{\text {pred }}=R_{\text {pred }}+(3 \times 1)+(2 \times 1)=7+3+2=12
$$


Now suppose that the required 12 units are not available and it is necessary to choose between staging three resources at the power substation and sending two units to the telecommunications node. In this case, the population and economic impact values could be used as weights to make the allocation decision. Note, however, that the allocation decision is assumed to be all-or-nothing, although it is rarely this way in the real world.

\section{Challenges using historical data}

This research began with the hypothesis that 911 call data would be an excellent foundation for a locally-specific and locally-driven decision support system. This hypothesis has been confirmed, albeit with a few caveats. The 911 data is the skeleton; putting flesh on it requires merging data from other sources. Working with historical data of any type has pitfalls such as legacy handling, missing data, incorrect values and unstructured text. In addition, information contained in 911 call data often includes HIPAA-sensitive information that must be removed.

A typical 911 entry includes a date and time stamp, jurisdiction responsible for event response, event category code, event latitude and longitude, and event ID; several other fields are administrative in nature. In the research presented here, continuation records enabled the derivation of other attributes such as the event duration, when the first unit was dispatched, when the unit arrived on the scene and the latitude and longitude (available as cross-street information), if these fields were empty in the main record. The records also included information from people at the scene, when mutual aid was called, the sources of the mutual aid, the sequence of activities that took place at the scene and when the event was closed.

Data from various public sites was also used [1, 21, 22]. The additional attributes included weather information (temperature, humidity, precipitation and wind speed) for the event date and time, demographic information about the geographical area, the number of vacant properties and the percentage of structures built before 1940. The vacancy and older structure attributes were identified by emergency management personnel as being relevant to the analysis of fire events.

Although the resulting list of attributes is fairly rich, important pieces are missing, including the specific equipment at the scene, the number of personnel in each responding unit, the times when the units left the scene and the decision process of the unit commander. In addition, some assumptions 
had to be made when analyzing the dataset. First, it was assumed that the incident duration is a proxy measurement for the size or scope of an event; small incidents should resolve more quickly than large ones. A related assumption was that the number of continuation records is correlated with the size of the event, because these records are essentially transcripts of the activities that took place at the scene.

Without exact information about the number and makeup of the responding unit crews, it was assumed that all crews had the same level of training and each unit carried a full crew. The number of responders in a full crew is, in general, locality-specific; however, it was assumed that crews comprised two individuals.

Another critical assumption was that the closing time stamp on a 911 record truly represents the end of the active response period corresponding to an event. However, an official often remains at the scene to complete paperwork long after the response units have left and the record remains open until all the personnel have left the site. Thus, the assumption yields a pessimistic calculation of incident duration. However, without specific data about the end of the active response phase, there is no other way to determine the duration and the scope.

When presenting this work to stakeholders, it was emphasized that changes to the data collection process would make future analyses easier and more seamless. For instance, adding metadata about weather systems would allow the linkage of disaster events with a severe weather event such as Superstorm Sandy, thus providing the ability to see a complete picture of the damage caused by a major regional event.

Privacy concerns also impact emergency event data and CI/KR information. The sensitivity of the two datasets implies that large-scale data analysis approaches using Hadoop or MapReduce would be more challenging, thus impacting the analyses of multi-year 911 datasets and complex $\mathrm{CI} / \mathrm{KR}$ repositories.

\section{Conclusions}

The results show that historical data can provide an adequate framework for a regional decision support system. Additions to the dataset, such as foundational weather/event metadata, specific unit and equipment types, responder skill inventories and crew size models, would support fine-grained 
and richer resource predictions. Some regions already collect such data and incorporating it into the base data would be fairly straightforward.

Historical emergency data enables a decision maker to benefit from previous experience in the form of an expected incident footprint and to focus attention on the deviations of the current event from previous patterns. Because new events can be added to the dataset, these variations become a part of the evolving system and strengthen its predictive ability.

The importance of the proposed methodology is its use of locally-specific historical data - both emergency event data and $\mathrm{CI} / \mathrm{KR}$ asset data - to build the foundation for decision support. This distinguishes the methodology from other approaches that rely on theoretical analyses or simulations. The effectiveness of the methodology is demonstrated using two major allocation scenarios. Moreover, although the methodology is developed for a mid-sized region, it is generalizable to any region.

The eventual goal of the research is to predict resource needs along with the incident timeline, allowing emergency managers to site and stage resources in a community and determine the appropriate mix of volunteer, professional and community responders. Future research will attempt to solve the very difficult issue of dealing with large datasets with stringent privacy requirements - in order for municipalities to build and use decision systems, they must be able to perform the initial analyses needed to generate incident footprints. Unfortunately, the resources needed to cleanse, analyze and update large emergency management datasets are typically beyond the capabilities of most communities and cloud services do not yet provide adequate data privacy. Another difficult problem that will be considered is the determination of the range of the multiplier $\beta$ using mathematical and empirical techniques.

The work described in this paper has focused primarily on the supportive role of historical data in emergency management. Completing the system development requires fusing real-time, heterogeneous data with historical scenarios as shown in Figure 1. The system must quickly recognize the specific incident footprint to use based on the incoming data and present recommendations to the emergency manager. Case-based reasoning will be investigated as a method for retrieving an appropriate footprint.

Decision support systems are primarily used in response situations, although their impact goes beyond the active phase of a disaster. Decisions made during a crisis strongly affect recovery time and ultimately influence the overall resilience of a community. Therefore, metrics that predict re- 
covery success and measure resilience should be incorporated into a decision support system. The metrics should include an assessment of the capabilities, vulnerabilities and maturities of the affected communities and their assets [20].

This paper has dealt only with physical events that are difficult to predict and manage. As disasters involving cyber-physical systems become increasingly probable, it is important to be prepared for them. Unfortunately, there is no cyber analog of 911 data and the overwhelmingly private ownership of critical infrastructure, including crucial sectors such as telecommunications, increases the complexity of the problem. Future research will explore approaches for dealing with this important problem.

\section{Acknowledgements}

The authors acknowledge the generous support of the LMI Research Institute's Academic Partnership Program and thank the members of the LMI Logistics Analysis Group for their guidance. This research was partially supported by the National Science Foundation under Grant No. DUE-1303269. The authors also acknowledge the ongoing support of the New York State Department of Homeland Security and Emergency Services, and the Rochester Urban Area Working Group. Special thanks are due to Mr. Fred Rion, Administrator, Monroe County Emergency Preparedness and to Mr. John Merklinger, Director, Monroe County Emergency Communications. Any opinions, findings and conclusions or recommendations expressed in this pa-

per are those of the authors and do not necessarily reflect the views of the organizations or individuals listed above.

IMPORTANT NOTE TO IJCIP TYPESETTERS: I have checked and edited the references in this paper myself. Please DO NOT MODIFY the references - except to add hyperlinks. Please contact the Journal Manager Ms. Ramya Vasudevan if you have any questions.

Professor Sujeet Shenoi, Editor-in-Chief, IJCIP

\section{References}

[1] Advameg, Welcome to City-Data, Carthage, Illinois (www.city-data. com), 2015. 
[2] J. Bolten, Memorandum for the Heads of Executive Departments and Agencies, Office of Management and Budget, Washington, DC (www.whitehouse.gov/sites/default/files/omb/memoranda/fy04/ m-04-15.pdf), June 17, 2014.

[3] D. Bryant, Rethinking OODA: Toward a modern cognitive framework of command decision making, Military Psychology, vol. 18(3), pp. 183-206, 2006.

[4] S. Cavallini, C. d'Alessandro, M. Volpe, S. Armenia, C. Carlini, E. Brein and P. Assogna, A system dynamics framework for modeling critical infrastructure resilience, in Critical Infrastructure Protection VIII, J. Butts and S. Shenoi (Eds.), Springer, Heidelberg, Germany, pp. 141$154,2014$.

[5] R. Chen, R. Sharman, H. Rao and S. Upadhyaya, Coordination in emergency response management, Communications of the ACM, vol. 51(5), pp. 66-73, 2008.

[6] CrisisMappers, CrisisMappers: The Humanitarian Technology Network (crisismappers.net), 2015.

[7] Department of Homeland Security, National Emergency Communications Plan: Urban Area Communications Key Findings and Recommendations, Washington, DC (www.dhs.gov/xlibrary/assets/necp_ goal_1_findings_accessible.pdf), 2011.

[8] Department of Homeland Security, Automated Critical Asset Management System (ACAMS), Washington, DC (www.dhs.gov/ automated-critical-asset-management-system-acams), 2015.

[9] Department of Homeland Security, Infrastructure Protection Gateway, Washington, DC (www.dhs.gov/ipgateway), 2015.

[10] Federal Emergency Management Agency, National Incident Management System, Washington, DC (www.fema.gov/ national-incident-management-system), 2015.

[11] S. French and M. Turoff, Decision support systems, Communications of the ACM, vol. 50(3), pp. 39-40, 2007. 
[12] J. Harrald and T. Jefferson, Shared situational awareness in emergency management mitigation and response, Proceedings of the Fortieth Annual Hawaii International Conference on System Sciences, 2007.

[13] V. Hristidis, S. Chen, T. Li, S. Luis and Y. Deng, Survey of data management and analysis in disaster situations, Journal of Systems and Software, vol. 83(10), pp. 1701-1714, 2010.

[14] M. Khouj, S. Sarkaria, C. Lopez and J. Marti, Reinforcement learning using Monte Carlo policy estimation for disaster mitigation, in Critical Infrastructure Protection VIII, J. Butts and S. Shenoi (Eds.), Springer, Heidelberg, Germany, pp. 155-172, 2014.

[15] S. Liu, A. Duffy, R. Whitfield and I. Boyle, Integration of decision support systems to improve decision support performance, Knowledge and Information Systems, vol. 22(3), pp. 261-286, 2010.

[16] Monroe County Government, Fire Districts and Fire Station Locations, Rochester, New York (www2.monroecounty.gov/files/gis/County\% 20Maps-2013/Fire_Districts\%202013.pdf), 2015.

[17] Y. Peng, Y. Zhang, Y. Tang and S. Li, An incident information management framework based on data integration, data mining and multicriteria decision making, Decision Support Systems, vol. 51(2), pp. 316327, 2011.

[18] L. Robbins and N. Kleinfield, Four firefighters shot, two fatally, in New York; gunman dead, New York Times, December 24, 2012.

[19] C. Romanowski and J. Schneider, Critical infrastructure protection and risk analysis in a mid-sized city, Proceedings of the IEEE Conference on Technologies for Homeland Security, pp. 517-522, 2012.

[20] J. Schneider, C. Romanowski, R. Raj, S. Mishra and K. Stein, Measurement of locality-specific resilience: An operational model, Proceedings of the IEEE International Symposium on Technologies for Homeland Security, 2015.

[21] Wikipedia, Wikipedia Homepage (www.wikipedia.org), 2015. 
[22] World Weather Online, World Weather Online Homepage, Manchester, United Kingdom (developer.worldweatheronline.com/page/ explorer-premium), 2015.

[23] L. Zheng, C. Shen, L. Tang, T. Li, S. Luis and S. Chen, Applying data mining techniques to address disaster information management challenges on mobile devices, Proceedings of the Seventeenth ACM SIGKDD International Conference on Knowledge Discovery and Data Mining, pp. 283-291, 2011. 